\title{
๖The Contribution of High-Frequency Wind-Generated Surface Waves to the Stokes Drift
}

\author{
LuC LENAIN ${ }^{\mathrm{a}}$ AND NiCK PIZZO ${ }^{\mathrm{a}}$

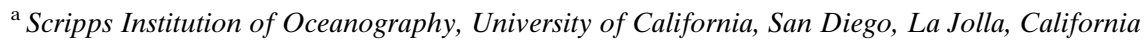

(Manuscript received 26 May 2020, in final form 27 August 2020)

\begin{abstract}
The effects of nonbreaking surface waves on upper-ocean dynamics enter the wave-averaged primitive equations through the Stokes drift. Through the resulting upper-ocean dynamics, Stokes drift is a catalyst for the fluxes of heat and trace gases between the atmosphere and ocean. However, estimates of the Stokes drift rely crucially on properly resolving the wave spectrum. In this paper, using state-of-the-art spatial measurements (in situ and airborne remote sensing) from a number of different field campaigns, with environmental conditions ranging from 2 to $13 \mathrm{~m} \mathrm{~s}^{-1}$ wind speed and significant wave height of up to $4 \mathrm{~m}$, we characterize the properties of the surface wave field across the equilibrium and saturation ranges and provide a simple parameterization of the transition between the two regimes that can easily be implemented in numerical wave models. We quantify the error associated with instrument measurement limitations, or incomplete numerical parameterizations, and propose forms for the continuation of these spectra to properly estimate the Stokes drift. Depending on the instrument and the sea state, predictions of surface Stokes drift may be underestimated by more than $50 \%$.
\end{abstract}

KEYWORDS: Atmosphere-ocean interaction; Currents; Wave breaking; Waves, oceanic; Wind waves; In situ oceanic observations

\section{Introduction}

Deep-water surface gravity waves play a crucial role in the marine boundary layer, modulating the exchange of mass, momentum, heat, and gases between the ocean and the atmosphere (Melville 1996; Cavaleri et al. 2012). Irrotational surface waves have particle orbits that are not closed, but instead are slightly elliptic, leading to a drift in their direction of wave propagation, known as Stokes drift. This drift is usually inferred from the directional surface wave spectrum (Kenyon 1969). Accurately estimating the Stokes drift is critical for a number of applications; from the study of upper ocean and airsea interaction processes, such as Langmuir circulations (Craik and Leibovich 1976; Leibovich 1983; McWilliams et al. 1997) that rely on a proper representation of the wave-induced drift (McWilliams and Restrepo 1999; Belcher et al. 2012), to the prediction of the transport of pollutants, oil spills and drifting objects (see also Lenain et al. 2019a). Additionally, better evaluation of the Stokes drift may lead to an improved predictive capability of larger-scale ocean dynamics (Shrira and Almelah 2020) that play a crucial role in weather and climate models (Breivik et al. 2019, among others).

In recent years, improvements in remote sensing and in situ observational techniques have led to significant progress in our ability to measure and understand spatiotemporal properties of surface gravity waves. In Lenain and Melville (2017), properties of the directional distribution of the surface wave field across the equilibrium and saturation ranges (Phillips 1985) were investigated from airborne lidar data (see also Melville et al. 2016). They demonstrated that the omnidirectional

¿ Denotes content that is immediately available upon publication as open access.

Corresponding author: Luc Lenain, llenain@ucsd.edu wavenumber spectra, $\phi(k)$, where $k$ is the wavenumber, exhibits a consistent power-law behavior, proportional to $k^{-5 / 2}$ in the equilibrium range and $k^{-3}$ in the saturation range, as predicted by Phillips (1985). These two regions of the wave spectrum have been extensively studied previously, both through theoretical analysis (see, e.g., Phillips 1958; Toba 1973; Kitaigorodskii 1983; Phillips 1958), spatial and temporal in situ observations (Donelan et al. 1985; Battjes et al. 1987; Hwang et al. 2000; Romero and Melville 2010a; Melville et al. 2016, among others) and numerical investigation (Pushkarev et al. 2003; Romero and Melville 2010b, among others) of the windgenerated wave field, but never with the broad spectral range required to fully capture and parameterize the transition from equilibrium to saturation ranges. The surface wave spectra dataset from Lenain and Melville (2017), along with recent observations from two other experiments described in the next section, offer a unique opportunity to carefully investigate the impact of spectral shape, and specifically the high-frequency surface wave contribution, to the total Stokes drift.

Mixing in the upper ocean controls the transfer of heat, and trace gases, between the atmosphere and ocean. The heat content serves as an important boundary condition for coupled air-sea models of both weather and climate. Errors in estimates of these fluxes can lead to biases in sea surface temperature. Through large-eddy simulations (LES), Li et al. (2016) recently showed that surface gravity waves enhance mixing in the ocean boundary layer, and therefore need to be better parameterized in numerical models ( $\mathrm{Li}$ et al. 2017). These predictions rely crucially on estimates of the turbulent Langmuir number, given by the ratio of the wind friction velocity to the Stokes drift. Therefore, it is critical to properly estimate the Stokes drift, which serves as the motivation for this paper.

Kenyon (1969), based on Phillips (1966), first related the wave energy spectrum to the Stokes drift. Since then, there has been considerable attention given to estimating the Stokes 
drift through a minimal number of environmental variables that characterize the wave spectrum, particularly in recent years (Breivik et al. 2016, 2014; van den Bremer and Breivik 2017). The integral computations are subtle, as the directional distribution of the waves crucially modulates the total Stokes drift (Webb and Fox-Kemper 2015), and one needs to resolve the small-scale waves, which significantly contribute to the drift (Pizzo et al. 2019). The directionality of the wave field, from the spectral peak to the realignment of the capillary waves with the longer gravity waves, is still a source of uncertainty, both in measurements and more severely in ocean wave models (Stopa et al. 2016; Liu et al. 2019). Furthermore, many studies employ the omnidirectional wave spectrum when computing the Stokes drift (McWilliams and Restrepo 1999; Sullivan et al. 2007; Breivik et al. 2014). The omnidirectional spectrum is also equivalent to the third spectral moment (see, e.g., Webb and Fox-Kemper 2015), and it has been recognized that the higherfrequency waves play an important role in properly computing this moment (Rascle et al. 2006; Webb and Fox-Kemper 2015; Sutherland and Melville 2015). In this paper, as a first step in fully constraining the Stokes drift, we focus on omnidirectional wave spectrum effects, while a manuscript on the directional effects is currently in preparation.

Here, in addition to the observations presented in Lenain and Melville (2017), we also consider measurements collected during two additional field programs, the Langmuir and Inner Shelf Office of Naval Research (ONR) Directed Research Initiative (DRI) field efforts (LCDRI2017 and ISDRI2017, respectively), providing a much broader range of environmental conditions which leads to a significantly improved parameterization of the transition between equilibrium and saturation ranges. This dataset provides a unique opportunity to characterize the contribution, across a broad range of scales, to the Stokes drift, and in turn, the error caused by the use of frequency-limited wave spectra or numerical wave spectra with an incorrect parameterization of the transition from equilibrium to saturation ranges.

\section{Experiments, instrumentation, and environmental conditions}

\section{a. Experiments}

This study is based on data collected during three ONR funded programs: SOCAL2013, LCDRI2017, and ISDRI2017. The first two projects were focused on phase-resolved measurements of wind and waves. Observations over a broad range of environmental conditions were collected. Both of these experiments were located between San Clemente and San Nicholas Islands (vicinity of $33^{\circ} 13.202^{\prime} \mathrm{N}, 118^{\circ} 58.767^{\prime} \mathrm{W}$ ) where the floating ocean research platform R/P FLIP was moored from 7 to 22 November 2013 and from 16 March to 10 April 2017, for the SOCAL2013 and LCDRI2017 experiments, respectively. R/P FLIP was instrumented with a suite of meteorological sensors to characterize the atmospheric, surface and subsurface conditions at the experiment site. Data from the ISDRI2017 experiment were collected from 5 to 21 September 2017 off the coast of Point Sal, California. In that case, surface conditions were estimated from an airborne lidar, as described in Lenain et al. (2019b). Overall, the environmental conditions considered here have wind speeds ranging from 2 to $13 \mathrm{~m} \mathrm{~s}^{-1}$, and significant wave height $H_{s}$ in the range of approximately 1 to $4 \mathrm{~m}$.

\section{b. The Modular Aerial Sensing System}

Spatiotemporal measurements of the sea surface topography and surface kinematics were collected from a Partenavia P68 aircraft that was instrumented with the Modular Aerial Sensing System (MASS), an instrument package developed at the Scripps Institution of Oceanography, as described in Melville et al. (2016). The instrument package is built around a Q680i waveform scanning lidar (Riegl, Austria), used to make spatiotemporal measurements of the sea surface elevation. The sensor has a maximum pulse repetition rate of $400 \mathrm{kHz}$, a maximum line scan rate of $200 \mathrm{~Hz}$, and has been used at altitudes up to $1500 \mathrm{~m}$ with sufficient lidar pulse returns for surface-wave measurements. All data collected are carefully georeferenced from the aircraft to an Earth-coordinate frame using a Novatel SPAN-LN200, a GPS-IMU system combining GPS technology with an inertial measurement unit (IMU) using fiber-optic gyros and solid-state accelerometers to provide position and attitude data at up to $200 \mathrm{~Hz}$. After postprocessing, we typically find absolute vertical errors of 2-4 cm (per lidar pulse) for the final topographic product [for more details, see Melville et al. (2016), Lenain and Melville (2017), and Lenain et al. (2019b)].

\section{c. Environmental conditions}

During the SOCAL2013 and LCDRI2017 experiments, a suite of atmospheric sensors were installed on R/P FLIP's port boom to characterize the marine atmospheric boundary layer variables that are used in our analysis. Although the setup was slightly different in each experiment [see technical details in Grare et al. (2018) and Lenain et al. (2019b)], the friction velocity in the air was computed from a sonic anemometer (Gill R3-50) mounted on a vertical mast that was deployed from the end of the horizontally extended 20-m-long port boom of FLIP in both experiments, using eddy correlation techniques. Here the friction velocity $u_{*}$ in the air is given by

$$
u_{*}=\left({\overline{u^{\prime} w^{\prime}}}^{2}+{\overline{v^{\prime} w^{\prime}}}^{2}\right)^{1 / 4}
$$

where $u, v$, and $w$ represent the three components of the wind vector in the along, cross, and vertical directions, respectively, and the prime denotes deviations from the mean. The covariances $\overline{u^{\prime} w^{\prime}}$ and $\overline{v^{\prime} w^{\prime}}$ are computed over 30 -min records.

During the ISDRI experiment, the environmental conditions were estimated remotely using the MASS. Here the friction velocity $u_{*}$ was computed using the method described in Lenain et al. (2019b).

\section{Spectral depiction of wind-generated surface waves across the equilibrium-saturation ranges}

Phillips (1985) proposed a model to describe the "equilibrium" range, based on the assumption of balance, proportionality and similar order of magnitude of the terms in the statistical equilibrium radiative transfer equation (viz., wave-wave interactions, 

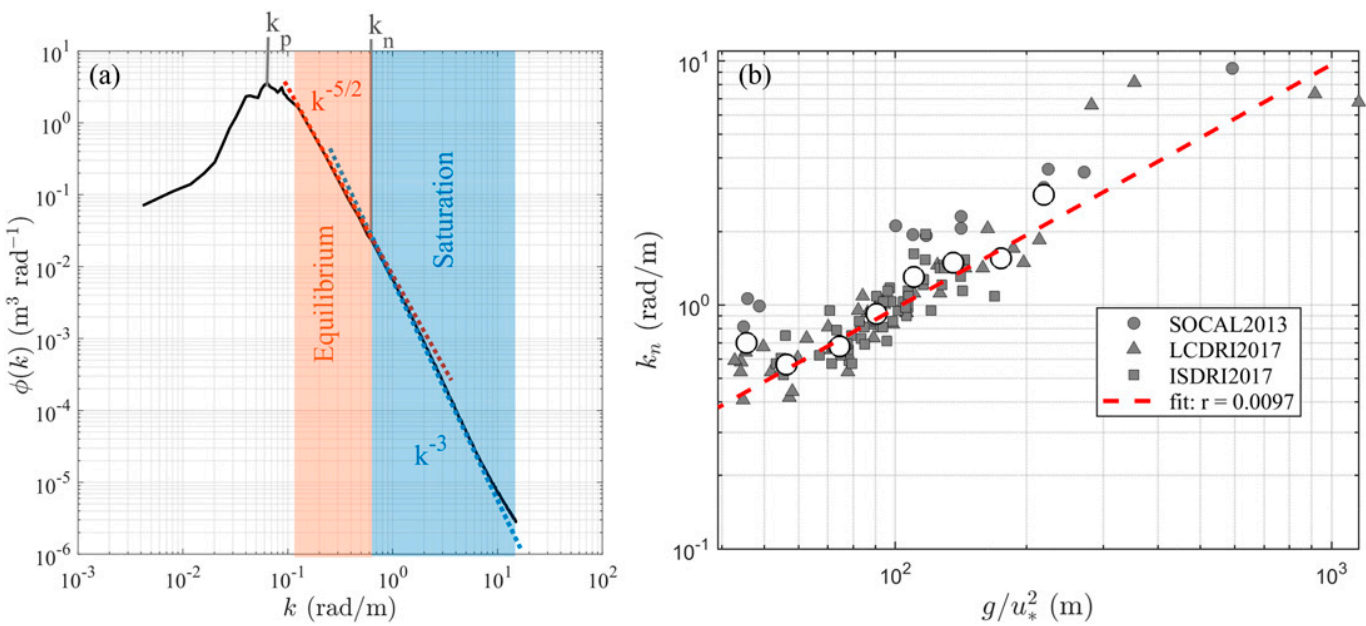

FIG. 1. (a) Example of the omnidirectional wavenumber spectrum collected on 15 Nov 2013 during the SOCAL2013 experiment using observations collected from an airborne topographic scanning lidar (Lenain and Melville 2017). Note the presence of both equilibrium and saturation ranges, showing both $-5 / 2$ and -3 spectral slopes over the three-decade bandwidth of the data. (b) Transition wavenumber $k_{n}$ plotted against $g / u_{*}^{2}$ for three experiments (SOCAL2013, LCDRI2017, and ISDRI2017). The Phillips (1985) parameter $r=k_{n} u_{*}^{2} / g$ is best fit to the data (red dashed line) and is equal to $r=9.7 \times 10^{-3}$. The white circles represent bin-averaged values.

wind forcing, and wave-breaking dissipation). Phillips's model predicts a $k^{-5 / 2}$ slope for the equilibrium range of the omnidirectional spectrum. Beyond the equilibrium range, spatial and temporal observations of wind waves show a power-law transition from a $k^{-5 / 2}$ to a $k^{-3}$ slope corresponding to another regime, the so-called saturation range (Forristall 1981; Banner 1990; Romero and Melville 2010a; Lenain and Melville 2017). In that case, the primary balance is between the wind input and the dissipation from breaking waves, as the time scales in this range are short enough such that nonlinear wave-wave interaction term becomes negligible. Observations of the transition between these two regimes is difficult, and our novel measurements over these ranges enabled this work.

\section{a. Methods}

Swaths of ocean topography collected from the MASS lidar were carefully georeferenced from the aircraft to an Earth-coordinate frame three-dimensional point cloud. For SOCAL2013 and LCDRI2017, 5-km-long swaths of data collected within $10 \mathrm{~km}$ of $\mathrm{R} / \mathrm{P}$ FLIP (where the atmospheric measurements were conducted) were gridded and interpolated on a regular grid, with the horizontal spatial resolution being a function of the flight altitude: $d x=d y=0.1 \mathrm{~m}$ for aircraft altitudes lower than $200 \mathrm{~m}$ above mean sea level (MSL), corresponding to a typical swath width of $50-150 \mathrm{~m}, d x=d y=0.2 \mathrm{~m}$ for altitudes ranging from 200 to $400 \mathrm{~m} \mathrm{MSL}$, and $d x=d y=$ $1 \mathrm{~m}$ for higher altitudes (with a corresponding swath width of $400-800 \mathrm{~m}$ ). The data collected along the cross-track edges of the swath were discarded because of high dropout rates ( $<10 \%-15 \%$ pulse returns). Two-dimensional fast Fourier transforms were computed over $5-\mathrm{km}$ segments with $50 \%$ overlap. All segments were first detrended, then tapered with a two-dimensional Hanning window and finally padded with zeros $(25 \%)$.
To correct for the Doppler shift induced by the relative motion between the phase speed of the wave and the aircraft velocity, each spectrum was corrected iteratively following the method developed by Walsh et al. (1985). The change in wavenumber component in the along-track direction is taken as

$$
\delta k_{x}=\omega / v_{a}
$$

where $\omega(k)\left(\operatorname{rads}^{-1}\right)$ is the radial wave frequency, computed from a deep-water dispersion relationship, and $v_{a}\left(\mathrm{~m} \mathrm{~s}^{-1}\right)$ is the aircraft velocity in the along-track direction.

A similar approach was taken for the data collected during the ISDRI2017 experiment. In that case, as the operational area included very shallow to deep water, we only considered water depth $h$ larger than $50 \mathrm{~m}$.

We next introduce the omnidirectional wave spectrum $\phi(k)$, defined as the azimuthally averaged directional spectrum,

$$
\phi(k)=\int_{0}^{2 \pi} F(k, \theta) k d \theta
$$

where $F(k, \theta)$ is the wave directional spectrum. Figure 1a shows an example of the azimuthally integrated omnidirectional spectrum computed from data collected during the SOCAL2013 experiment. The variable $k_{p}$ represents the spectral peak wavenumber of the wind-generated waves. The separation at wavenumber $k_{n}$ of the spectral slopes into -2.5 (equilibrium) and -3 (saturation) regions is clear, and in this case the transition wavenumber $k_{n}$ is found to be equal to $0.6 \mathrm{rad} \mathrm{m}^{-1}$.

\section{b. Improved parameterization of the equilibrium-saturation range transition}

Part of the analysis presented in Lenain and Melville (2017) was dedicated to the characterization and parameterization of the transition wavenumber $k_{n}$. We expanded their work to 
include two additional field experiments, LCDRI2017 and ISDRI2017. For each azimuthally integrated spectrum, the transition wavenumber $k_{n}$ was computed by estimating the intersection between a $k^{-5 / 2}$ fit in the equilibrium range and a constant saturation value at higher wavenumbers, that is, $k^{-3}$. Results are presented in Fig. 1b, where the transition wavenumber is plotted against $g / u_{*}^{2}$, a quantity introduced in Phillips (1985) to describe the upper end of the equilibrium spectrum such that $r$ (sometimes referred to as Phillips's constant), a constant, is defined as

$$
r=k_{n} u_{*}^{2} / g
$$

Here we find $r=9.7 \times 10^{-3}$.

This result is of interest to the wave modeling community. While there has been growing recognition of the existence of equilibrium and saturation regimes, properly parameterizing their transition has been a challenge (Liu et al. 2019). Here we corroborate the parameterization proposed by Phillips (1985) for the transition from equilibrium to saturation ranges that only requires the friction velocity $u_{*}$ and therefore can be easily implemented in operational wave models.

\section{Stokes drift}

The Stokes drift is computed from the directional spectrum $F$, given by (Kenyon 1969)

$$
\mathbf{U}_{s}=2 \iint F(\mathbf{k}) \sqrt{g k} e^{2 k z} \mathbf{k} d \mathbf{k},
$$

where $k=|\mathbf{k}|$ and $z$ is the depth (i.e., $z=0$ at the surface).

Here, the Stokes drift magnitude $U_{s}(z)$ based on the omnidirectional wave spectrum $\phi(k)$ is defined as

$$
U_{s}(z)=2 \int_{k_{p}}^{\infty} \phi(k) \sqrt{g k} e^{2 k z} k d k,
$$

where $k_{p}$ is the peak wavenumber of the wind-waves, $z$ is the depth, and $\phi(k)$ is the omnidirectional wave spectrum defined in Eq. (3). Hence, the spectral shape of surface waves will have a direct impact on Stokes drift. Note, following Breivik et al. (2014) and Pizzo et al. (2019), we ignore the contribution to the Stokes drift of the very low wavenumbers (i.e., swell), as these waves are not steep so that their contribution to the total drift is very small, and since the focus in this study is on wind-generated waves.

Following Eq. (6), the Stokes drift is computed for all three experiments described in the previous section, and at seven set depths $z$ : 0 (surface), $-0.1,-0.2,-0.5,-1,-2$, and $-5 \mathrm{~m}$. Since the transition between saturation and equilibrium ranges are clearly characterized in the three datasets, we can compute the contribution of the equilibrium range to the total windgenerated surface Stokes drift, where the Stokes drift in the equilibrium range is defined as

$$
U_{s, \mathrm{eq}}(z)=2 \int_{k_{p}}^{k_{n}} \phi(k) \sqrt{g k} e^{2 k z} k d k .
$$

This is shown in Fig. 2, for $z=0 \mathrm{~m}$ (surface), plotted against the friction velocity $u_{*}$. We find that as the friction velocity

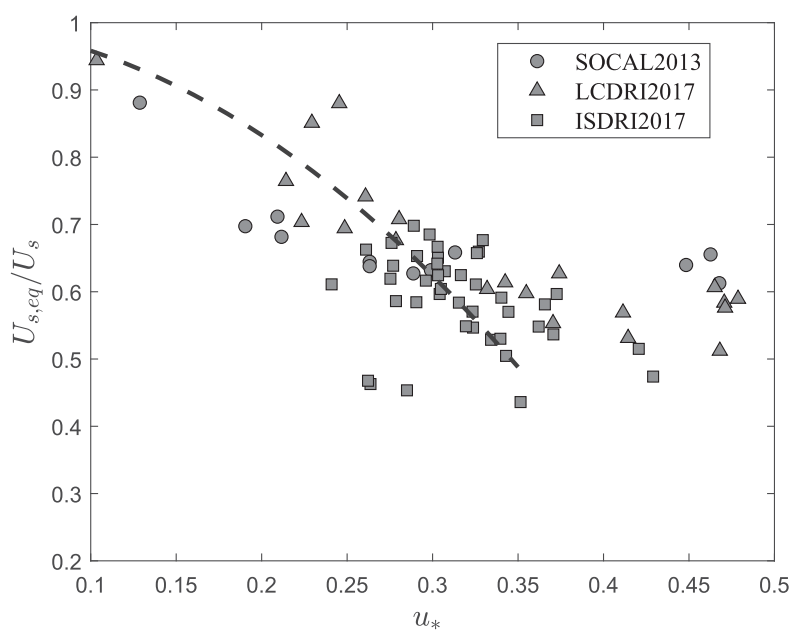

FIG. 2. Contribution of the equilibrium range to the total surface Stokes drift $U_{s}(z=0 \mathrm{~m})$. Here the Stokes drift is computed from Eqs. (6) and (7). As the friction velocity increases, the contribution of the equilibrium range decreases, reaching a plateau for $u_{*}$ larger than $0.35 \mathrm{~m} \mathrm{~s}^{-1}$. A fit to the data (dashed line) is also shown, such that $u_{s, \mathrm{eq}} / u_{s}=1-\alpha u_{*}^{2}$, where $\alpha=4.18$.

increases, the contribution of the equilibrium range decreases, reaching a plateau for $u_{*}$ larger than $0.35 \mathrm{~m} \mathrm{~s}^{-1}$, with a value of approximately $45 \%-65 \%$ of the total Stokes drift. In other words, the contribution from the high-frequency part of the surface wave spectrum, i.e., the saturation range, is not negligible when computing Stokes drift, especially at the surface, and needs to be fully resolved.

\section{Contribution of the high-frequency wind-generated surface waves}

In this section, we look at the impact of the cutoff frequency on the magnitude of the Stokes drift, effectively highlighting the significance of the contribution from the higher wavenumber part of the saturation spectra. This is particularly relevant, as Stokes drift is often computed using surface wave measurements without paying much attention to the frequency or wavenumber spectral range, and in particular the maximum frequency resolved.

For reference, directional wave buoys are generally able to resolve surface waves up to scales of approximately $0.5-0.6 \mathrm{~Hz}$, similar to what global reanalysis products, such as the ERA datasets (e.g., ERA-Interim or ERA5) from ECMWF, can now resolve (Uppala et al. 2005; Dee et al. 2011). It is clear from Fig. 1 that such cutoff frequencies (wave buoys: $k_{c}=$ $1.35 \mathrm{rad} \mathrm{m}^{-1}$; ERA5: $k_{c}=3.8 \mathrm{rad} \mathrm{m}^{-1}$ ) are not adequate to resolve the Stokes drift contribution from the saturation range.

To quantify the errors associated with the use of surface wave spectra that do not resolve high enough frequencies to accurately compute Stokes drift, we introduce here $U_{s, \mathrm{nb}}$, such that

$$
U_{s, \mathrm{nb}}(z)=2 \int_{k_{p}}^{k_{c}} \phi(k) \sqrt{g k} e^{2 k z} k d k,
$$

where $k_{c}$ is a cutoff frequency. 


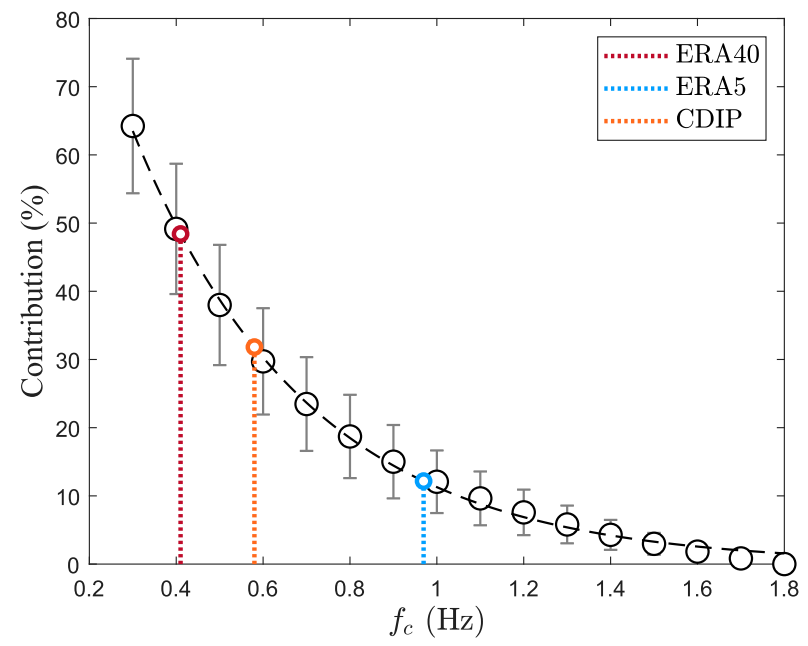

FIG. 3. Bin-averaged surface Stokes drift contribution of the shorter waves as a function of cutoff frequency $f_{c}$ computed from the surface wave spectra collected during all three experiments. Also shown are the current cutoff frequencies for wave spectral products, ECMWF ERA-40, ERA5, and CDIP (observations), and error bars for each bin of frequency $(0.1 \mathrm{~Hz})$.

We compute the contribution (i.e., underestimation) from the shorter waves as a function of cutoff frequency $f_{c}$ in estimating $U_{s}$ such that

$$
\text { contribution }=100 \times \frac{\left|U_{s}(z)-U_{s, \mathrm{nb}}(z)\right|}{U_{s}(z)} .
$$

Figure 3 shows the contribution of the shorter waves to the surface $(z=0)$ Stokes drift defined in Eq. (9) computed for cutoff frequencies $f_{c}$ ranging from 0.3 to $1.8 \mathrm{~Hz}$ (i.e., from 0.36 to $13 \mathrm{rad} \mathrm{m}^{-1}$ ). We find the contribution rapidly decreases as $f_{c}$ increases, following an exponential decay (dashed line), such that

$$
\text { contribution }=a e^{-b f_{c}},
$$

where $a$ is equal to 133.15 and $b$, the $e$-folding scale, is 2.47 , estimated through a least-squares fit $\left(r^{2}=0.99\right)$. This simple relationship can be used to correct surface Stokes drift estimates computed from spectrally limited in situ observations or reanalysis products.

For reference, the cutoff frequency of commonly used spectral wave products is also shown, the ECMWF ERA- $40^{1}$ and ERA5 reanalysis global datasets (Uppala et al. 2005; Dee et al. 2011), and buoy-based observations from the Coastal Data Information Program (CDIP) network (https://cdip. ucsd.edu/). We find that computing Stokes drift from these products alone would lead to significant underestimations, ranging from approximately 50\% error for ERA-40, 34\% for the CDIP wave products, down to $10 \%-15 \%$ for ERA5.

Moreover, it is sometimes assumed that the high-frequency part of the surface wave field does not contribute to the Stokes

\footnotetext{
${ }^{1}$ The reanalysis product used in Belcher et al. (2012).
}

(a)

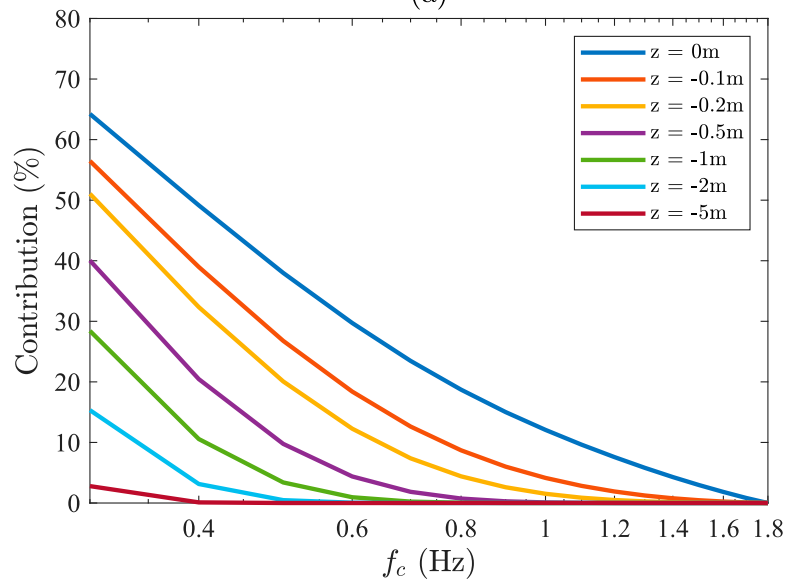

(b)

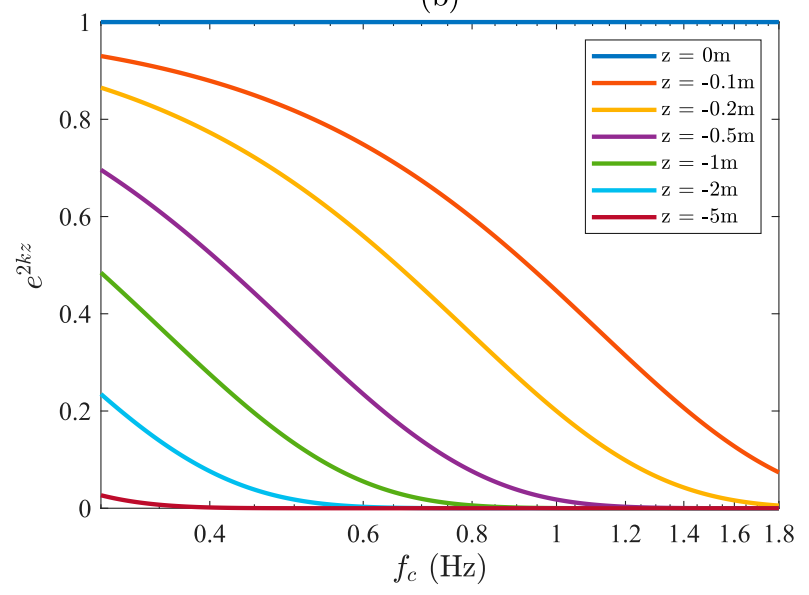

FIG. 4. (a) Stokes drift contribution of the shorter waves as a function of cutoff frequency $f_{c}$ and depth (down to $-5 \mathrm{~m}$ ) computed from the spectra of surface wave data collected during the three experiments. (b) Depth dependence of the Stokes drift $e^{2 k_{c} z}$ plotted against cutoff frequency. Below 5-m depth, the contribution to the Stokes drift from waves of frequencies larger than $0.4 \mathrm{~Hz}$ is negligible.

drift at depth, even close to the surface. This is investigated in Fig. 4a, where the Stokes drift error is shown for depths ranging from the surface down to $5 \mathrm{~m}$. As expected, as depth increases, the contribution of the shorter waves to the Stokes drift is reduced. At $5 \mathrm{~m}$ depth, we find that the contribution from waves of frequencies larger than $0.4 \mathrm{~Hz}$ is negligible. Nevertheless, and this is of importance for upper-ocean modeling, the contribution from shorter waves-of frequencies larger than, for example, in situ observations $(0.4-0.5 \mathrm{~Hz}$ at best)-is not negligible above $5 \mathrm{~m}$ depth and increases rapidly closer to the surface. Note, Clarke and Van Gorder (2018) recently proposed a wave breaking frequency $\left(\approx 8 \mathrm{rad} \mathrm{m}^{-1}\right)$ as an upper integral limit for spectral Stokes drift computation. Here we find that such approach would be reasonable for depths larger than $0.5 \mathrm{~m}$. It would, however, lead to an underestimation of the Stokes drift for shallower depths, up to approximately $15 \%$ 
at the surface. Figure $4 \mathrm{~b}$ shows the depth-dependent term of the Stokes drift in Eq. (7), $e^{2 k z}$, plotted against cutoff frequency, another way of illustrating the penetration depth of short waves and their contribution to the total Stokes drift. Finally, we note that although the contribution of the short waves to the Stokes drift attenuates rapidly with depth, their shear values are large, so that we expect them to be an important contribution to the turbulent kinetic energy budget [see, e.g., Eq. (1) of Belcher et al. (2012)].

Ultimately, this result provides guidance on the contribution of high-frequency surface waves to horizontal wave induced transport in the upper ocean, particularly near the ocean surface.

\section{Is adding a spectral tail to limited bandwidth spectra sufficient?}

An approach to mitigating the availability of limitedbandwidth wave spectra when computing directional Stokes drift has been to add a high-frequency spectral tail of set slope (i.e., $f^{-5}$ or $k^{-3}$ ) to the spectrum (see, e.g., Belcher et al. 2012), or extrapolating a wave spectrum to a set saturation level (Romero et al. 2012). While the use a parametric tail when calculating Stokes drift via a wave model is now more common, there has yet to be a consensus on the best approach. At the surface, the third spectral moment can be used to calculate the omnidirectional Stokes drift, which would automatically include any parametric tail employed by the model. Recently, it has become more common to calculate the full directional Stokes drift and patch it with an omnidirectional tail (which does not account for directional effects). At depth, various profiles have been used with different tails. For reference, unlike ECWAM, the wave model used for ERA5, WAVEWATCH III does not include a parametric tail (or at least until version 4.18) in its default output for the surface directional Stokes drift.

Here we attempt to evaluate how well the application of a simple parametric tail works using the broad bandwidth wave spectra that were collected during the three field programs with the MASS lidar instrument.

In Fig. 5, we compare the intentionally frequency-limited estimate of the surface Stokes drift $U_{s, \mathrm{nb}}(z=0)$, where $k_{c}$ is set here to $0.67 \mathrm{rad} \mathrm{m}^{-1}$ (i.e., $f_{c}=0.41 \mathrm{~Hz}$ ), corresponding to the cutoff frequency of ERA-40 used in Belcher et al. (2012), to the "true" Stokes drift (red dots), computed from the full omnidirectional spectra collected during the three experiments. ${ }^{2}$ The dashed line represents 1:1, and the white circles represent bin-averaged values. As discussed in the prior section, the need for including high frequencies in the computation of the Stokes drift is obvious here, as we found underestimation of close to $50 \%$ at times when they were not included.

Following Belcher et al. (2012), we also applied a saturation tail $\left(f^{-5}\right.$ or $\left.k^{-3}\right)$ to the frequency-limited spectra for frequencies larger than $k_{c}$ (gray dots, Fig. 5). While the Stokes drift that was estimated using the $k^{-3}$ tail show good agreement for large $U_{s}$, we nevertheless find that this approach underestimates the

\footnotetext{
${ }^{2}$ For reference, the maximum wavenumber resolved in the field observations is approximately $13 \mathrm{rad} \mathrm{m}^{-1}$.
}

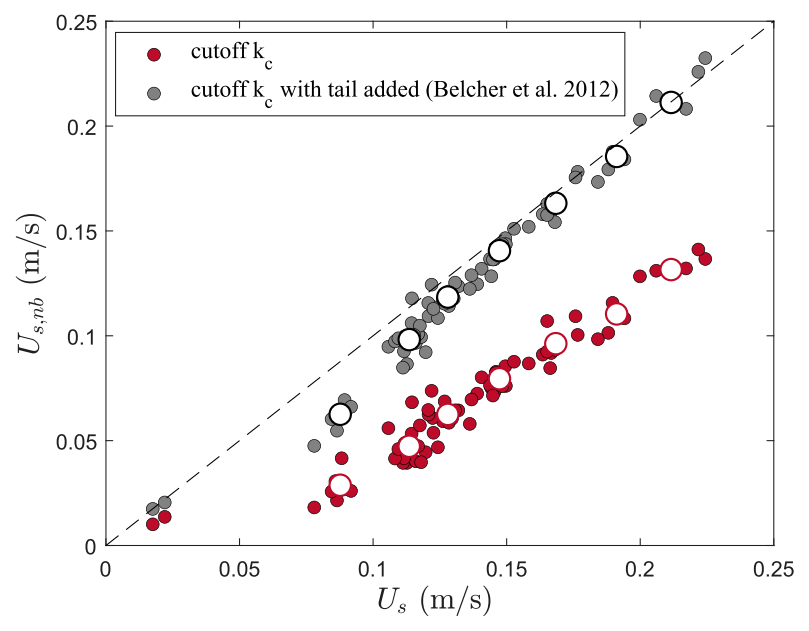

FIG. 5. The surface Stokes drift computed spectrally using a cutoff wavenumber $k_{c}=0.67 \mathrm{rad} \mathrm{m}^{-1}$, the highest ECMWF ERA-40 resolved frequency (red), and taking a similar approach to Belcher et al. (2012), in which they patched a saturation tail $\left(f^{-5}\right.$ or $k^{-3}$ ) for frequencies larger than $k_{c}$, plotted against the "true" Stokes drift $U_{s}$ computed over the complete wavenumber range of the measured spectra. Here all three experiments are considered. The dashed line represents 1:1, and the white circles represent binaveraged values. While the $U_{s}$ estimates when patching a $k^{-3}$ tail show good agreement for large $U_{s}$ (this is expected, since $k_{n}<k_{c}$ in that case) we find that this approach underestimates the Stokes drift by $10 \%-30 \%$ for smaller $U_{s}$, in the $0.075-0.15 \mathrm{~m} \mathrm{~s}^{-1}$ range. Last, the need for patching a tail when wave spectra that are computed explicitly do not resolve the high wavenumbers is obvious.

surface Stokes drift by $10 \%-30 \%$ for smaller values of $U_{s}$, in the $0.075-0.15 \mathrm{~m} \mathrm{~s}^{-1}$ range. This brings up the importance of properly characterizing the spectral shape of the wave spectrum described in an earlier section. As shown in Fig. 6, depending on the cutoff frequency $k_{c}$ relative to the transition wavenumber $k_{n}$, applying a set slope tail to the spectra will have very different outcomes. When $k_{c}>k_{n}$, we find the surface Stokes drift to be properly estimated. However, when $k_{c}<$ $k_{n}$, the transition from equilibrium to transition regimes effectively is forced to $k_{c}$, in turn truncating the contribution of the high-frequency part of the wave spectrum to the Stokes drift, as highlighted in Fig. 6.

\section{Errors caused by the misrepresentation of the transition between the equilibrium and saturation ranges}

Misrepresentation of the transition between the equilibrium and saturation ranges is another potential source of errors when computing Stokes drift. To characterize this effect, we make use of an updated version of the model [see Eq. (A3)] of surface Stokes drift from Pizzo et al. (2019). The model has been validated with field observations, showing remarkable agreement with the estimates computed from observed wave spectra using Eq. (6), as described in the appendix. Here we use the model over the range of environmental parameters observed during the three experiments, and artificially vary the 

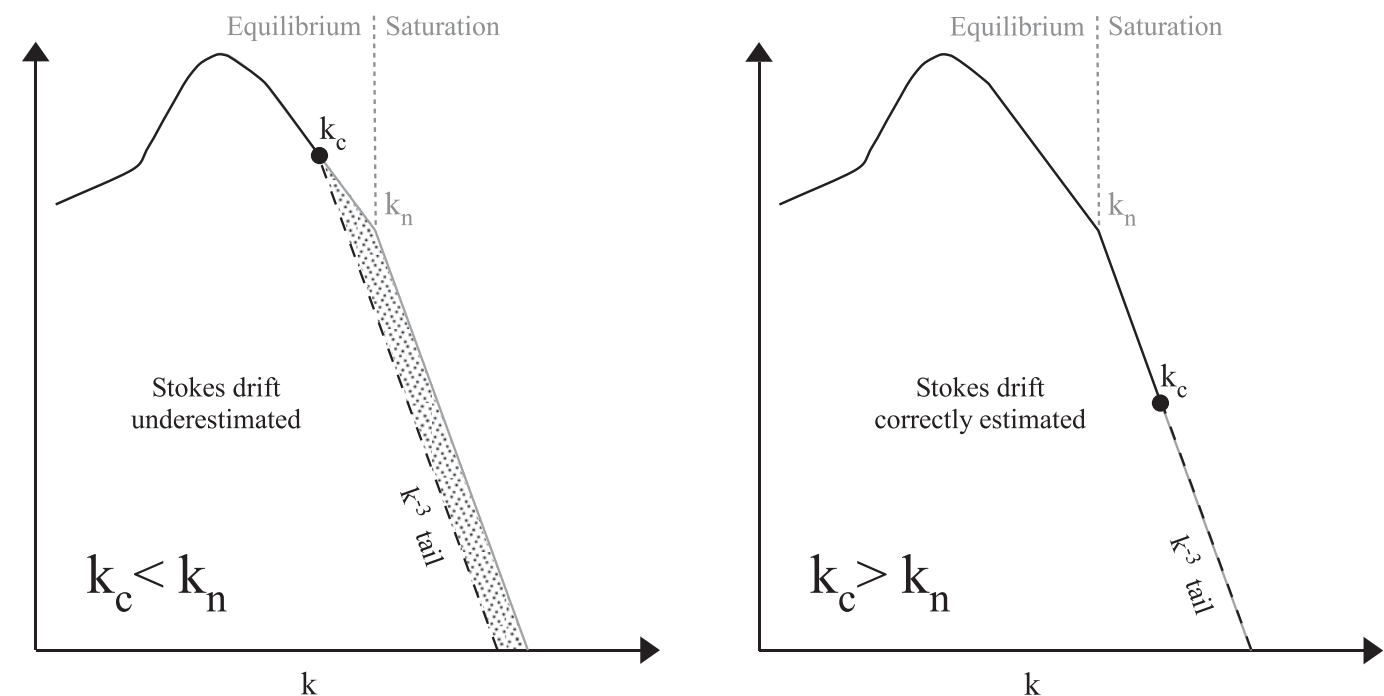

FIG. 6. Impact of adding a tail to limited bandwidth wave spectrum: (left) $k_{c}<k_{n}$ and (right) $k_{c}>k_{n}$.

transition wavenumber $k_{n}$, defined here as $k_{n \text {,est }}$, and compare the resulting Stokes drift to the one computed with the accurate $k_{n}$. Results are shown in Fig. 7. This demonstrates the need to pay particular attention to the spectral shape of the surface wave spectra used in Stokes drift computations. For example, a factor-of-2 underestimation of $k_{n}$ leads to a $15 \%$ error in surface Stokes drift estimate, which is significant.

\section{A practical example: Stokes drift and turbulent Langmuir number from in situ buoy measurements}

To highlight the findings presented in the prior sections, we used publicly available data collected from a NDBC station located in the Gulf of Mexico (42040). This buoy is equipped with both wind and surface wave measurement capabilities and is located at $29.208^{\circ} \mathrm{N}, 88.226^{\circ} \mathrm{W}$. More details about this station can be found on the NDBC website (https:// www.ndbc.noaa.gov/station_page.php?station=42040).

Here, the surface Stokes drift is computed in three different ways. The first way uses the original, limited bandwidth surface wave spectrum provided by NDBC $(f=0.02-0.485 \mathrm{~Hz})$ - that is, no corrections applied. Also used are two other versions that include a high-frequency spectral tail: one case in which an $f^{-5}$ saturation tail is added for $f>0.485 \mathrm{~Hz}$, and a second version in which the spectra are patched with an equilibrium $f^{-4}$ (or $k^{-5 / 2}$ ) and saturation $f^{-5}$ (or $k^{-3}$ ) tails for cases in which the transition frequency $f_{n}=\left(g k_{n}\right)^{1 / 2} /(2 \pi)$ is larger than $0.485 \mathrm{~Hz}$. for the latter, $k_{n}$ is computed using Eq. (4) with $r$ taken as $9.7 \times 10^{-3}$. The maximum frequency of the high-frequency tail $f_{M}=$ $\left(g k_{M}\right)^{1 / 2} /(2 \pi)$ is defined as the cutoff wavenumber above which the directional wave spectrum is assumed isotropic, based on the findings of Lenain and Melville (2017), such that $k_{M}=\left(g / u_{*}^{2}\right) \exp \left[\left(\pi / 2-\theta_{0}\right) / \gamma\right]$, where $\theta_{0}=2.835$ and $\gamma=0.48$ [see Eq. (4) of Lenain and Melville (2017) for details].

Results are presented in Fig. 8, showing data collected from NDBC 42040 from April 2017 through January 2018. Figure 8a shows the wind speed collected at $z=3.8 \mathrm{~m}$ from the buoy, and Fig. $8 \mathrm{~b}$ shows the surface Stokes drift, as described above.
As expected from the previous sections, we find the Stokes drift to be significantly underestimated when no spectral tail is added. We also find that properly parameterizing the transition from equilibrium to saturation ranges in the spectral tail also has significant impact, particularly for $U_{s}$ smaller than $0.1 \mathrm{~m} \mathrm{~s}^{-1}$. This is highlighted in Fig. 8c, where the Stokes drift estimates are shown over a shorter period of time (September 2017). The two estimates with spectral tail added collapse for higher winds (in that case $f_{n}<f_{c}=0.485 \mathrm{~Hz}$ ), around 10 September 2017, whereas significant differences are found as the wind decreases, after 13 September 2017.

Next, recall that upper-ocean mixing is parameterized through the turbulent Langmuir number (McWilliams et al. 1997), defined as

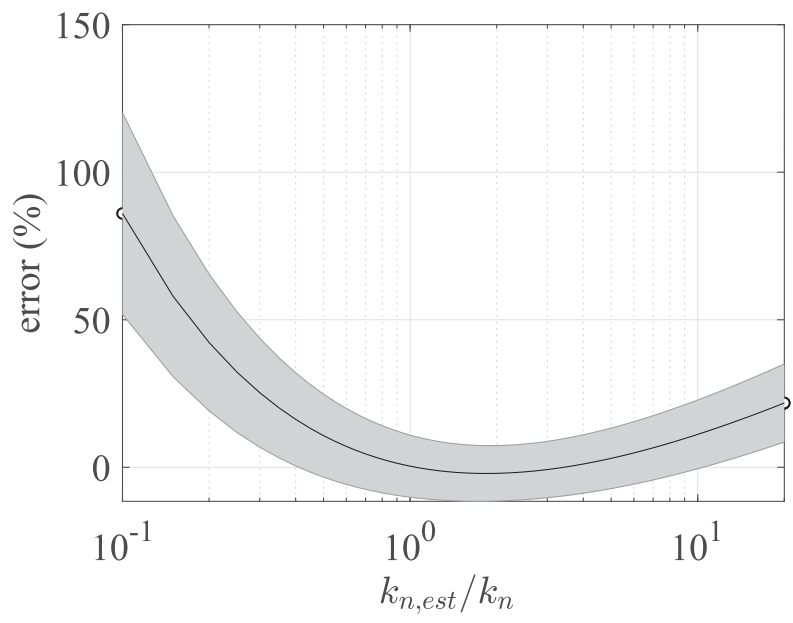

FIG. 7. Effect of the transition wavenumber $k_{n}$ on the total Stokes drift, combining the three available datasets. Here, $k_{n}$ is the measured transition wavenumber and $k_{n \text {,est }}$ is the one used to compute $U_{s}$ from Eq. (A3). Errors are computed relative to the "true" Stokes drift, computed from the azimuthally integrated spectrum $\phi(k)$. 
(a)

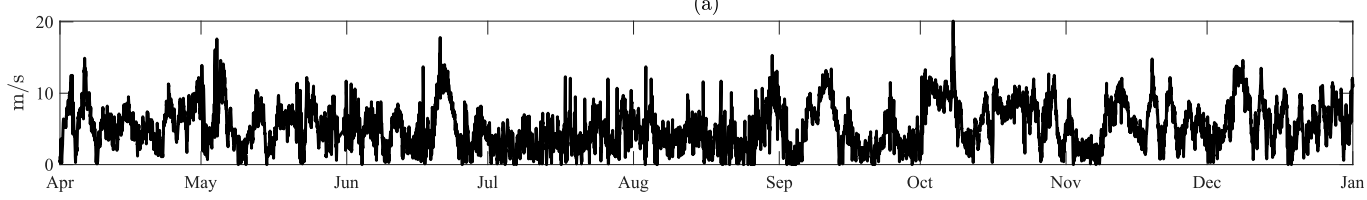

(b)

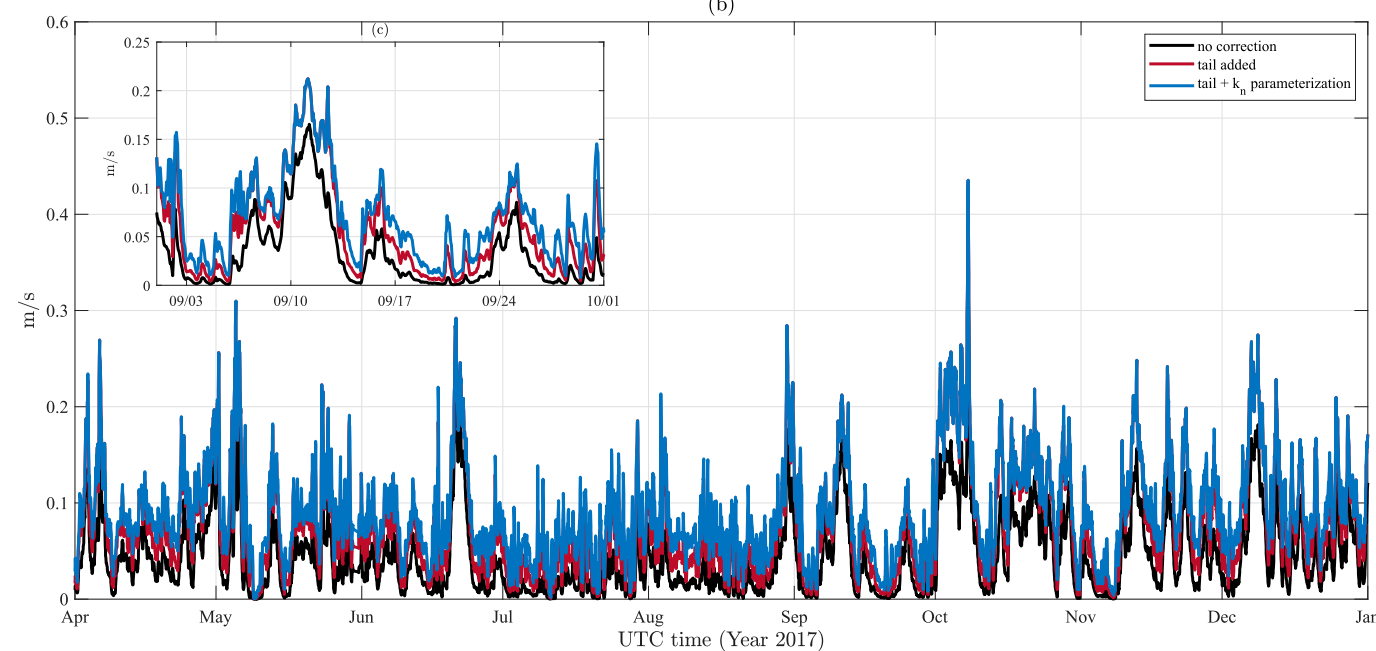

FIG. 8. (a) Wind speed $\left(\mathrm{m} \mathrm{s}^{-1}\right)$ collected from NDBC buoy 42040 in the Gulf of Mexico in 2017. (b) Surface Stokes drift, computed using the measured wave spectra, along with two products that include a high-frequency spectral tail: the red line shows the surface Stokes drift with an $f^{-5}$ saturation tail, and the blue one is patched with an equilibrium $f^{-4}$ and saturation $f^{-5}$ tails for cases in which the wave spectrum cutoff frequency is lower than $f_{n}=$ $\left(g k_{n}\right)^{1 / 2} /(2 \pi)$. In that latter case, $k_{n}$ is computed using Eq. (4) with $r=9.7 \times 10^{-3}$. (c) A shorter length of the record, highlighting the significant differences in Stokes drift magnitude between each version. Also note that, as expected from the previous section, the two products with tail added collapse for higher winds, around 10 Sep 2017, and significant differences are found as the wind decreases, after 13 Sep 2017.

$$
\mathrm{La}_{t}=\left(u_{*_{w}} / u_{s}\right)^{1 / 2},
$$

where $u_{* w}$ is the friction velocity in the water such that $u_{*_{w}}=$ $\left(\tau \rho_{w}\right)^{1 / 2}$, where $\rho_{w}$ is the water density and $\tau$ is the surface stress. As this parameter is used in both weather and climate models to parameterize mixing and the heat content in the upper ocean, it is critical to ensure that this quantity is computed correctly. Figure 9 shows the turbulent Langmuir number computed for the data presented in Fig. 8c. As anticipated, the addition of a spectral tail significantly reduces $\mathrm{La}_{t}$. What is less expected is the sensitivity of the turbulent Langmuir number to the shape of the spectral tail. Specifically, we find that if the transition frequency between equilibrium and saturation ranges is not parameterized correctly, $\mathrm{La}_{t}$ can be overestimated by up to $30 \%-40 \%$, which may lead to significant biases in sea surface temperatures ( $\mathrm{Li}$ et al. 2017). Note, other nondimensional Langmuir numbers have been proposed as alternatives to the turbulent Langmuir number, such as the surface layer-averaged Langmuir number (Harcourt and D'Asaro 2008) and the projected Langmuir number (Van Roekel et al. 2012). We expect that the impact of models for Stokes drift of high-frequency waves on these Langmuir numbers will be less than for the turbulent Langmuir number $\mathrm{La}_{t}$ as in the former the Stokes drift is averaged over a finite upper-ocean layer.

\section{Discussion}

In this paper, we provide a better description of the spectral evolution of wind-generated waves. Specifically, we expand the work of Lenain and Melville (2017) on the partitioning into equilibrium and saturation ranges of surface gravity waves, as originally proposed by Phillips (1985), using high-resolution measurements of wind-generated surface gravity waves. In particular, we propose a simple parameterization of the transition from equilibrium to saturation regimes of windgenerated surface gravity waves, only requiring the atmospheric friction velocity $u_{*}$ as input, that could be readily implemented in wave models. This is significant, as currently most operational models do not explicitly parameterize this transition (Liu et al. 2019).

Error analysis was performed to quantify the errors in the estimated Stokes drift, as a function of cutoff frequency and transition wavenumber. It is found that there might be significant underestimation (exceeding 50\%) in estimates of Stokes drift based on instrument or reanalysis product limitations. Important is that we provide an explanation for why this occurs and offer a means of correcting Stokes drift when only spectrally limited data is available. We identify that the misrepresentation of the transition from equilibrium to saturation ranges has an impact on the estimate of Stokes drift computed spectrally. While the analysis is mostly focused here on surface 


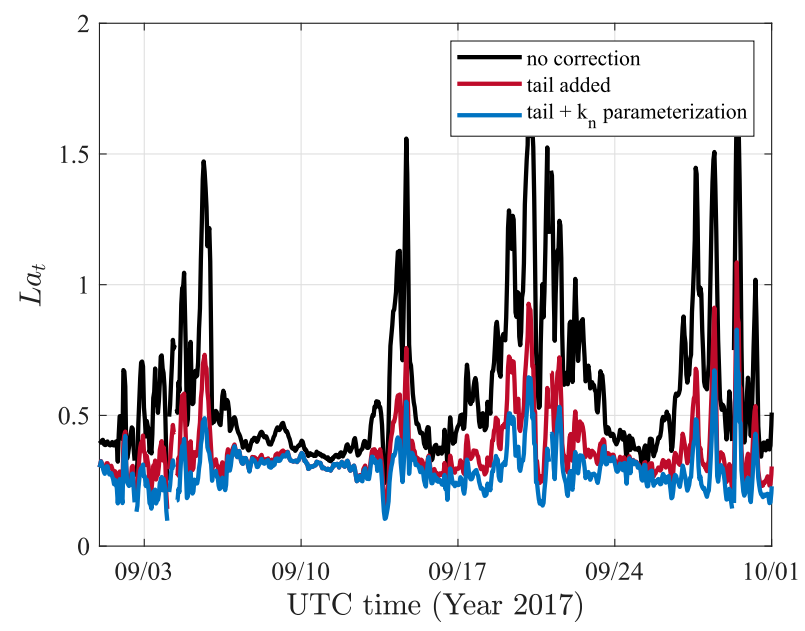

FIG. 9. Turbulent Langmuir number $\mathrm{La}_{t}$ for the same time period that is displayed in Fig. 8c. Note the significant differences found among the three versions and the significant overestimation of $\mathrm{La}_{t}$ when using the original, noncorrected surface wave spectrum from NDBC.

Stokes drift, where we anticipate the contribution of the shorter waves to be largest, depth dependence is also investigated, to provide guidance on the contribution of surface waves to horizontal transport in the upper ocean, near the ocean surface.

The Stokes drift plays a crucial role in upper-ocean dynamics, via interactions with existing vorticity through the socalled vortex force term (Leibovich 1983). This mixes the upper ocean and sets the boundary conditions for coupled airsea models. Estimates of the mixing is often parameterized through the Langmuir number, a ratio of the wind friction velocity to the Stokes drift. As this parameter is used in both weather and climate models, it is crucial to have high-fidelity observations of this quantity. The work done in this paper provides better estimates of the Stokes drift, and hence better estimates of the turbulent Langmuir number to be used in these coupled models. The sensitivity of this number to the spectral estimate of Stokes drift was demonstrated here.

The directionality of the wave field is still a source of uncertainty both in measurements and more significantly in ocean wave models, and is the focus of ongoing studies. As the wave field becomes bimodal above the spectral peak, over a specific range of wavenumbers, we expect the omnidirectional spectra overestimates the drift, consistent with Webb and Fox-Kemper (2015). However, this estimate is very sensitive to the directional spreading function, and needs a more detailed examination, which is currently underway by the present authors. Nevertheless, the emphasis in the present work on the need to include high-frequency waves, and to properly parameterize the equilibrium-saturation range transition in limitedbandwidth wave products also directly applies to directional surface wave estimates of the Stokes drift.

Acknowledgments. The authors gratefully acknowledge numerous discussions with Ken Melville on this topic prior to his death in autumn 2019. The authors are grateful to Aspen Helicopter for providing flight resources. We thank Laurent Grare for providing the atmospheric data collected from R/P FLIP and for helpful discussions and Nick Statom for his support during the field experiments and in the analysis of the lidar data. We are thankful to two anonymous reviewers for their suggestions, which improved the paper. This research was supported by grants from the Physical Oceanography programs at ONR (Grants N00014-17-1-2171, N00014-14-1-0710, N00014-15-1-2871, N00014-19-1-2635, and N00014-17-13005), NSF (OCE; Grant OCE-1634289), and NASA (Grant 80NSSC19K1688).

Data availability statement. All presented data are available at the UCSD Library Digital Collection (https://library.ucsd. $\mathrm{edu} / \mathrm{dc})$.

\section{APPENDIX}

\section{Stokes Drift Model}

Here we revisit the model of the Stokes drift from Pizzo et al. (2019) to validate it using field observations of the directional wave spectrum collected during three experiments (SOCAL2013, LCDRI2017, ISDRI2017). In addition to the model considered there, we add a high wavenumber maximum, $k_{M}$, above which we assume the waves do not contribute to the Stokes drift. The maximum wavenumber $k_{M}$ is defined as the cutoff wavenumber above which the directional wave spectrum is assumed isotropic, based on the findings of Lenain and Melville (2017), such that $k_{M}=\left(g / u_{*}^{2}\right) \exp \left[\left(\pi / 2-\theta_{0}\right) / \gamma\right]$, where $\theta_{0}=2.835$ and $\gamma=0.48$ [see Eq. (4) of Lenain and Melville (2017) for details]. As the Stokes drift goes like $\phi(k) k^{3 / 2}$, particular care is needed in where to place this cutoff (Breivik et al. 2014); this topic is discussed in more detail below.

With this addition, following Pizzo et al. (2019), the Stokes drift $U_{s}$ at the surface $(z=0)$ can be shown to scale as

$$
U_{s}=\beta u_{*} \ln \left(\frac{r g}{u_{*}^{2}} \frac{1}{k_{p}}\right)+4 B\left(\frac{u_{*}}{r^{1 / 2}}-\sqrt{\frac{g}{k_{M}}}\right),
$$

where $B$ is the saturation constant, given a saturation spectrum $B k^{-3}$ (see Fig. 1), set to $7 \times 10^{-3}$ in our study, based on the findings of Lenain and Melville (2017), and $\beta$ is an empirical parameter, often referred to as Toba's constant (Toba 1973), that can be computed directly from the equilibrium range of wind generated surface waves such that

$$
\phi(k)=\frac{\beta}{2} \frac{u_{*}}{\sqrt{g}} k^{-5 / 2}
$$

While it is defined here as a constant, Resio et al. (2004) and Romero and Melville (2010a) introduced a weak dependence of $\beta$ on the wave phase speed at the spectral peak, and effective wave age, respectively.

Figure A1a shows the modeled surface Stokes drift computed from Eq. (A1) in comparison with the Stokes drift computed explicitly using the measured omnidirectional spectra as described in Eq. (7) for $z=0$. The dashed line 
(a)

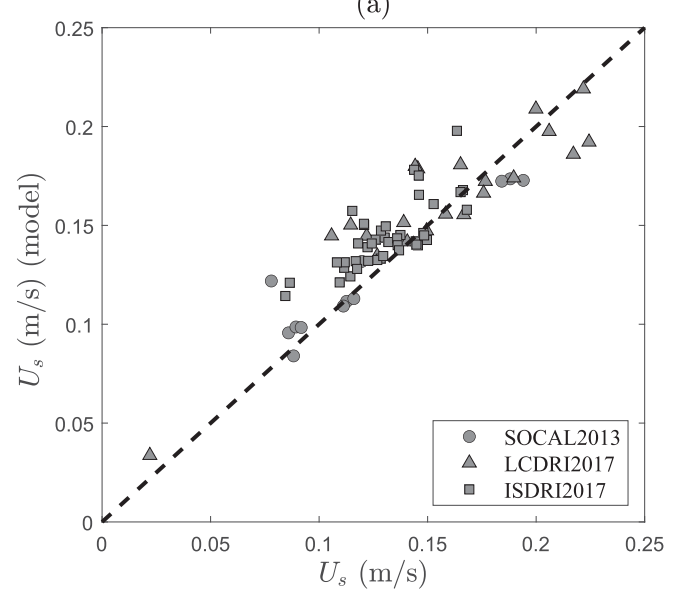

(b)

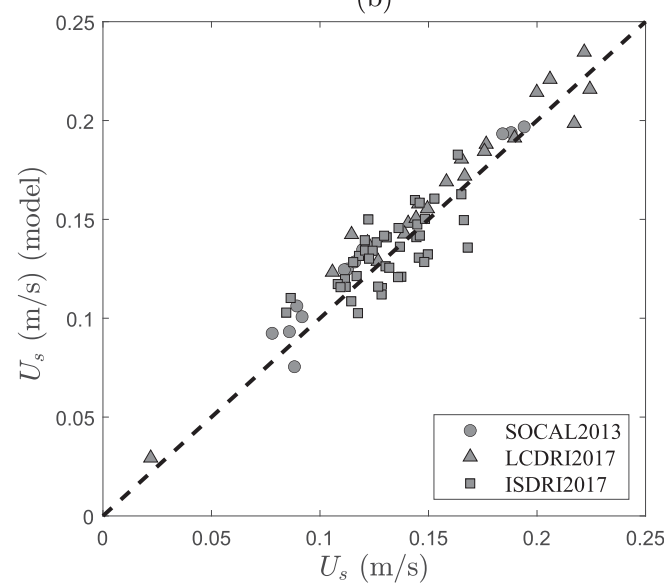

FIG. A1. Surface Stokes drift computed from (a) Eq. (A1) or (b) Eq. (3), plotted against the Stokes drift computed from the azimuthally integrated spectra [Eq. (7)] for the three experiments considered here. The dashed line represents $1: 1$. We see that there is good agreement between the model and the full prediction of the Stokes drift.

shows a 1:1 ratio. We find good agreement between the model and measured Stokes drift, with a coefficient of determination $R^{2}$ of 0.78 . Note that by setting Toba's constant to 0.105 , and in turn to avoid the need for a measurement of the compensated wave spectrum or another parameterization for this variable, we find, not unexpectedly, more scatter but nevertheless a reasonable agreement with $R^{2}=0.58$.

Next, following Pizzo et al. (2019), we can rewrite Eq. (A1) in terms of the spectrally weighted phase velocity $c_{\text {pm }}$ such that

$$
U_{s}=\beta u_{*} \ln \left(2 r \frac{c_{\mathrm{pm}}^{2}}{u_{*}^{2}}\right)+4 B\left(\frac{u_{*}}{r^{1 / 2}}-\sqrt{\frac{g}{k_{M}}}\right) .
$$

Here $c_{\mathrm{pm}}$ is defined following Sutherland and Melville (2015), in an attempt to better represent the wind-wave portion of the spectrum, because describing a broad, wind generated wave field only using a peak frequency has significant limitations (Lenain and Melville 2017). Results are presented in Fig. A1b; we find a very good agreement between the proposed model and the surface Stokes drift computed from the wave spectra, with an $R^{2}$ value of 0.88 , much better than what was found using Eq. (A1).

Note that there is a factor of 2 missing in the drift estimates of Pizzo et al. (2019), which is now corrected in Eqs. (A1) and (A3). This did not affect their scaling relationships, because an arbitrary constant was involved in each of the distinct regimes (e.g., equilibrium and saturation ranges).

\section{REFERENCES}

Banner, M. L., 1990: Equilibrium spectra of wind waves. J. Phys. Oceanogr., 20, 966-984, https://doi.org/10.1175/1520-0485(1990) 020<0966:ESOWW>2.0.CO;2.

Battjes, J., T. Zitman, and L. Holthuusen, 1987: A reanalysis of the spectra observed in JONSWAP. J. Phys. Oceanogr., 17, 1288-1295, https://doi.org/10.1175/1520-0485(1987)017<1288:AROTSO> 2.0.CO;2.
Belcher, S. E., and Coauthors, 2012: A global perspective on Langmuir turbulence in the ocean surface boundary layer. Geophys. Res. Lett., 39, L18605, https://doi.org/10.1029/ 2012GL052932.

Breivik, Ø., P. A. Janssen, and J.-R. Bidlot, 2014: Approximate Stokes drift profiles in deep water. J. Phys. Oceanogr., 44, 2433-2445, https://doi.org/10.1175/JPO-D-14-0020.1.

—, J.-R. Bidlot, and P. A. Janssen, 2016: A Stokes drift approximation based on the Phillips spectrum. Ocean Modell., 100, 49-56, https://doi.org/10.1016/j.ocemod.2016.01.005.

_- A. Carrasco, J. Staneva, A. Behrens, A. Semedo, J.-R. Bidlot, and O. J. Aarnes, 2019: Global Stokes drift climate under the RCP8. 5 scenario. J. Climate, 32, 1677-1691, https://doi.org/ 10.1175/JCLI-D-18-0435.1.

Cavaleri, L., B. Fox-Kemper, and M. Hemer, 2012: Wind waves in the coupled climate system. Bull. Amer. Meteor. Soc., 93, 1651-1661, https://doi.org/10.1175/BAMS-D-11-00170.1.

Clarke, A. J., and S. Van Gorder, 2018: The relationship of nearsurface flow, Stokes drift and the wind stress. J. Geophys. Res. Oceans, 123, 4680-4692, https://doi.org/10.1029/2018JC014102.

Craik, A., and S. Leibovich, 1976: A rational model for Langmuir circulations. J. Fluid Mech., 73, 401-426, https://doi.org/ 10.1017/S0022112076001420.

Dee, D. P., and Coauthors, 2011: The ERA-Interim reanalysis: Configuration and performance of the data assimilation system. Quart. J. Roy. Meteor. Soc., 137, 553-597, https://doi.org/ 10.1002/qj.828.

Donelan, M. A., J. Hamilton, and W. Hui, 1985: Directional spectra of wind-generated waves. Philos. Trans. Roy. Soc. London, 315A, 509-562, https://doi.org/10.1098/rsta.1985.0054.

Forristall, G. Z., 1981: Measurements of a saturated range in ocean wave spectra. J. Geophys. Res., 86, 8075-8084, https://doi.org/ 10.1029/JC086iC09p08075.

Grare, L., L. Lenain, and W. K. Melville, 2018: Vertical profiles of the wave-induced airflow above ocean surface waves. J. Phys. Oceanogr., 48, 2901-2922, https://doi.org/10.1175/JPO-D-180121.1.

Harcourt, R. R., and E. A. D'Asaro, 2008: Large-eddy simulation of Langmuir turbulence in pure wind seas. J. Phys. Oceanogr., 38, 1542-1562, https://doi.org/10.1175/2007JPO3842.1. 
Hwang, P. A., D. W. Wang, E. J. Walsh, W. B. Krabill, and R. N. Swift, 2000: Airborne measurements of the wavenumber spectra of ocean surface waves. Part I: Spectral slope and dimensionless spectral coefficient. J. Phys. Oceanogr., 30, 2753-2767, https://doi.org/10.1175/1520-0485(2001)031<2753: AMOTWS $>2.0 . \mathrm{CO} ; 2$.

Kenyon, K. E., 1969: Stokes drift for random gravity waves. J. Geophys. Res., 74, 6991-6994, https://doi.org/10.1029/ JC074i028p06991.

Kitaigorodskii, S., 1983: On the theory of the equilibrium range in the spectrum of wind-generated gravity waves. J. Phys. Oceanogr., 13, 816-827, https://doi.org/10.1175/1520-0485(1983) $013<0816$ :OTTOTE $>2.0 . \mathrm{CO} ; 2$.

Leibovich, S., 1983: The form and dynamics of Langmuir circulations. Annu. Rev. Fluid Mech., 15, 391-427, https://doi.org/ 10.1146/annurev.fl.15.010183.002135.

Lenain, L., and W. K. Melville, 2017: Measurements of the directional spectrum across the equilibrium saturation ranges of wind-generated surface waves. J. Phys. Oceanogr., 47, $2123-$ 2138, https://doi.org/10.1175/JPO-D-17-0017.1.

—_, N. Pizzo, and W. K. Melville, 2019a: Laboratory studies of Lagrangian transport by breaking surface waves. J. Fluid Mech., 876, R1, https://doi.org/10.1017/jfm.2019.544.

—, N. M. Statom, and W. K. Melville, 2019b: Airborne measurements of surface wind and slope statistics over the ocean. J. Phys. Oceanogr., 49, 2799-2814, https://doi.org/10.1175/ JPO-D-19-0098.1.

Li, Q., A. Webb, B. Fox-Kemper, A. Craig, G. Danabasoglu, W. G. Large, and M. Vertenstein, 2016: Langmuir mixing effects on global climate: Wavewatch iii in cesm. Ocean Modell., 103, 145-160, https://doi.org/10.1016/j.ocemod.2015.07.020.

— B. Fox-Kemper, Ø. Breivik, and A. Webb, 2017: Statistical models of global Langmuir mixing. Ocean Modell., 113, 95114, https://doi.org/10.1016/j.ocemod.2017.03.016.

Liu, Q., W. E. Rogers, A. V. Babanin, I. R. Young, L. Romero, S. Zieger, F. Qiao, and C. Guan, 2019: Observation-based source terms in the third-generation wave model WAVEWATCH III: Updates and verification. J. Phys. Oceanogr., 49, 489-517, https://doi.org/10.1175/JPO-D-18-0137.1.

McWilliams, J. C., and J. M. Restrepo, 1999: The wave-driven ocean circulation. J. Phys. Oceanogr., 29, 2523-2540, https:// doi.org/10.1175/1520-0485(1999)029<2523:TWDOC >2.0.CO;2.

_ - P. P. Sullivan, and C.-H. Moeng, 1997: Langmuir turbulence in the ocean. J. Fluid Mech., 334, 1-30, https://doi.org/10.1017/ S0022112096004375.

Melville, W. K., 1996: The role of surface wave breaking in air-sea interaction. Annu. Rev. Fluid Mech., 28, 279-321, https:// doi.org/10.1146/annurev.fl.28.010196.001431.

—, L. Lenain, D. R. Cayan, M. Kahru, J. P. Kleissl, P. Linden, and N. M. Statom, 2016: The modular aerial sensing system. J. Atmos. Oceanic Technol., 33, 1169-1184, https://doi.org/ 10.1175/JTECH-D-15-0067.1.

Phillips, O. M., 1966: The Dynamics of the Upper Ocean. Cambridge University Press, $261 \mathrm{pp}$.

_ 1985: Spectral and statistical properties of the equilibrium range in wind-generated gravity waves. J. Fluid Mech., 156, 505-531, https://doi.org/10.1017/S0022112085002221.

- 1958: The equilibrium range in the spectrum of windgenerated waves. J. Fluid Mech., 4, 426-434, https://doi.org/ 10.1017/S0022112058000550.
Pizzo, N., W. K. Melville, and L. Deike, 2019: Lagrangian transport by nonbreaking and breaking deep-water waves at the ocean surface. J. Phys. Oceanogr., 49, 983-992, https://doi.org/ 10.1175/JPO-D-18-0227.1.

Pushkarev, A., D. Resio, and V. Zakharov, 2003: Weak turbulent approach to the wind-generated gravity sea waves. Physica $D$, 184, 29-63, https://doi.org/10.1016/S0167-2789(03)00212-4.

Rascle, N., F. Ardhuin, and E. A. Terray, 2006: Drift and mixing under the ocean surface: A coherent one-dimensional description with application to unstratified conditions. J. Geophys. Res., 111, C03016, https://doi.org/10.1029/2005JC003004.

Resio, D. T., C. E. Long, and C. L. Vincent, 2004: Equilibriumrange constant in wind-generated wave spectra. J. Geophys. Res., 109, C01018, https://doi.org/10.1029/2003JC001788.

Romero, L., and W. K. Melville, 2010a: Airborne observations of fetch-limited waves in the Gulf of Tehuantepec. J. Phys. Oceanogr., 40, 441-465, https://doi.org/10.1175/2009JPO4127.1.

, and $-2010 \mathrm{~b}$ : Numerical modeling of fetch-limited waves in the Gulf of Tehuantepec. J. Phys. Oceanogr., 40, 466-486, https://doi.org/10.1175/2009JPO4128.1.

,$- \ldots$, and J. M. Kleiss, 2012: Spectral energy dissipation due to surface wave breaking. J. Phys. Oceanogr., 42, 1421-1444, https://doi.org/10.1175/JPO-D-11-072.1.

Shrira, V. I., and R. B. Almelah, 2020: Upper-ocean Ekman current dynamics: A new perspective. J. Fluid Mech., 887, A24, https:// doi.org/10.1017/JFM.2019.1059.

Stopa, J. E., F. Ardhuin, A. Babanin, and S. Zieger, 2016: Comparison and validation of physical wave parameterizations in spectral wave models. Ocean Modell., 103, 2-17, https://doi.org/10.1016/j.ocemod.2015.09.003.

Sullivan, P. P., J. C. McWilliams, and W. K. Melville, 2007: Surface gravity wave effects in the oceanic boundary layer: Large-eddy simulation with vortex force and stochastic breakers. J. Fluid Mech., 593, 405-452, https://doi.org/10.1017/ S002211200700897X.

Sutherland, P., and W. K. Melville, 2015: Field measurements of surface and near-surface turbulence in the presence of breaking waves. J. Phys. Oceanogr., 45, 943-965, https:// doi.org/10.1175/JPO-D-14-0133.1.

Toba, Y., 1973: Local balance in the air-sea boundary processes. J. Oceanogr. Soc. Japan, 29, 209-220, https://doi.org/10.1007/ BF02108528.

Uppala, S. M., and Coauthors, 2005: The ERA-40 Re-Analysis. Quart. J. Roy. Meteor. Soc., 131, 2961-3012, https://doi.org/ 10.1256/QJ.04.176.

van den Bremer, T., and Ø. Breivik, 2017: Stokes drift. Philos. Trans. Roy. Soc. London, A376, 20170104, https://doi.org/ 10.1098/RSTA.2017.0104.

Van Roekel, L., B. Fox-Kemper, P. Sullivan, P. Hamlington, and S. Haney, 2012: The form and orientation of Langmuir cells for misaligned winds and waves. J. Geophys. Res. Oceans, 117, C05001, https://doi.org/10.1029/2011JC007516.

Walsh, E., D. Hancock III, D. Hines, R. Swift, and J. Scott, 1985: Directional wave spectra measured with the surface contour radar. J. Phys. Oceanogr., 15, 566-592, https://doi.org/10.1175/ 1520-0485(1985)015<0566:DWSMWT>2.0.CO;2.

Webb, A., and B. Fox-Kemper, 2015: Impacts of wave spreading and multidirectional waves on estimating Stokes drift. Ocean Modell., 96, 49-64, https://doi.org/10.1016/j.ocemod.2014.12.007. 